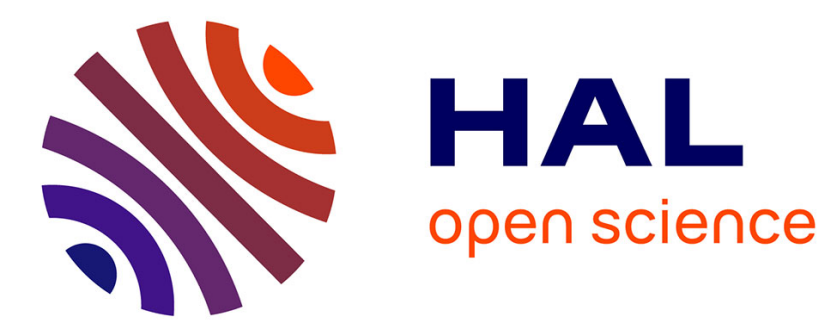

\title{
Sparse-Based Morphometry: Principle and Application to Alzheimer's Disease
}

Pierrick Coupé, Charles Deledalle, Charles H Dossal, Michèle Allard

\section{To cite this version:}

Pierrick Coupé, Charles Deledalle, Charles H Dossal, Michèle Allard. Sparse-Based Morphometry: Principle and Application to Alzheimer's Disease. Patch-Based Techniques in Medical Imaging (MICCAI), Oct 2016, Athènes, Greece. 10.1007/978-3-319-47118-1_6 . hal-01398763

\section{HAL Id: hal-01398763 https://hal.science/hal-01398763}

Submitted on 17 Nov 2016

HAL is a multi-disciplinary open access archive for the deposit and dissemination of scientific research documents, whether they are published or not. The documents may come from teaching and research institutions in France or abroad, or from public or private research centers.
L'archive ouverte pluridisciplinaire HAL, est destinée au dépôt et à la diffusion de documents scientifiques de niveau recherche, publiés ou non, émanant des établissements d'enseignement et de recherche français ou étrangers, des laboratoires publics ou privés. 


\title{
Sparse-Based Morphometry: Principle and Application to Alzheimer's Disease
}

\author{
Pierrick Coupé ${ }^{1,2}$, Charles-Alban Deledalle ${ }^{3,4}$, Charles Dossal ${ }^{3,4}$, Michèle \\ Allard $^{5,6,7}$, and the Alzheimer's Disease Neuroimaging Initiative* \\ 1 CNRS, LaBRI, UMR 5800, PICTURA, F-33400 Talence, France. \\ 2 Univ. Bordeaux, LaBRI, UMR 5800, PICTURA, F-33400 Talence, France. \\ 3 Univ. Bordeaux, IMB, UMR 5251, F-33400 Talence, France. \\ 4 CNRS, IMB, UMR 5251, F-33400 Talence, France. \\ 5 Univ. Bordeaux, INCIA, UMR 5287, F-33400 Talence, France. \\ ${ }^{6}$ CNRS, INCIA, UMR 5287, F-33400 Talence, France. \\ 7 EPHE, Bordeaux, France.
}

\begin{abstract}
The detection of brain alterations is crucial for understanding pathophysiological or neurodegenerative processes. The Voxel-Based Morphometry (VBM) is one of the most popular methods to achieve this task. Based on the comparison of local averages of tissue densities, VBM has been used in a large number of studies. Despite its numerous advantages, VBM is based on a highly reduced representation of the local brain anatomy since complex anatomical patterns are reduced to local averages of tissue probabilities. In this paper, we propose a new framework called Sparse-Based Morphometry (SBM) to better represent local brain anatomies. The presented patch-based approach uses dictionary learning to detect anatomical pattern modifications based on their shape and geometry. In our validation, the impact of the patch and group sizes is evaluated. Moreover, the sensitivity of SBM along Alzheimer's Disease (AD) progression is compared to VBM. Our results indicate that SBM is more sensitive than VBM on small groups and to detect early anatomical modifications caused by AD.
\end{abstract}

Keywords: voxel-based morphometry, Alzheimer's Disease, dictionary learning, abnormality detection, patch-based processing.

\section{Introduction}

In neurological diseases such as Alzheimer's Disease (AD), numerous studies suggest that some structures are affected by atrophy whereas others are relatively preserved. These brain abnormalities reflect pathophysiological processes, and are correlated with cognitive impairments and clinical symptoms. Thus, the

\footnotetext{
* Data used in preparation of this article were obtained from the Alzheimer's Disease Neuroimaging Initiative (ADNI) database (adni.loni.usc.edu). As such, the investigators within the ADNI contributed to the design and implementation of ADNI and/or provided data but did not participate in analysis or writing of this report. A complete listing of ADNI investigators can be found at: http://adni.loni.usc.edu/wp-content/uploads/how_to_apply/ADNI_Acknowledgement_List.pdf
} 
analysis of the anatomical brain integrity in patients is an important challenge of current research in neurology. Automatic detection of such brain abnormalities enables us to link anatomical substratum to cognitive performance or clinical symptoms. One of the classical methods used for automatic analysis of brain structures is voxel-based morphometry (VBM) [1]. VBM is an image processing framework enabling the identification of differences in the tissue density over the whole brain without a priori definition of a region of interest. Since its introduction, VBM has been successfully used in studies dedicated to a large variety of disorders. Despite its numerous advantages, VBM is based on a highly reduced representation of the local brain anatomy. Indeed, the complex local gray matter (GM) pattern is reduced to a local weighted average of GM probabilities through a Gaussian smoothing. While the importance of this smoothing step is well-known from a practical point of view [2], the use of a local average to represent complex anatomical patterns should be questioned. In this paper, we investigate the possibility of using a patch-based strategy to better represent the local brain anatomy. Recently, patch-based methods have demonstrated high performance in many neuroimaging applications such as anatomical structure segmentation [3]4 or automatic diagnosis [5]6. As recently proposed for segmentation purposes [4, we use a dictionary learning process to construct a sparse representation of anatomical patterns. Unlike 4, this sparse modeling of the brain anatomy is used to detect abnormalities through a new framework called Sparse-Based Morphometry (SBM). As shown later, SBM is especially well-suited to detect local modifications in terms of their shape and geometry. However, the inherent patch normalization involved in sparse-coding approaches makes SBM insensitive to modifications of the mean GM density within a patch. Therefore, we propose to combine the capability of VBM to detect modifications of local average GM density with the high ability of SBM to capture subtle alterations of the local shape of GM patterns. As discussed in the following, VBM can be viewed as the comparison of the patch mean while SBM compares the patch geometry. The contribution of this paper is threefold: (i) we present the new concept of SBM susceptible to better represent the complexity of local anatomical patterns, (ii) we propose to combine VBM and SBM in order to simultaneously analyze modifications of the local mean GM density and alterations of the shape and geometry of local GM patterns, and (iii) we evaluate the proposed framework on the ADNI dataset.

\section{Methods and Materials}

Subjects selection and Preprocessing In this study we used a sample of the standardized ADNI1 collection 7. By using the 818 baseline MRI scan at $1.5 \mathrm{~T}$, we created three groups of cognitively normal subjects $(\mathrm{CN})$, one group of patients with $\mathrm{AD}$, one group of subjects with Mild Cognitive Impairment (MCI) who progressed to $\mathrm{AD}$ (progressive MCI or $\mathrm{pMCI}$ ) and one group of subjects with MCI who remained stable during the follow-up period (sMCI). All the groups have the same number of subjects $(N=70)$. In addition, to remove potential 
Table 1: Demographic information about the considered groups

\begin{tabular}{|c|c|c|c|c|}
\hline Group & Size & Gender ( $\%$ Female) & Age (SD) & MMSE (SD) \\
\hline $\mathrm{CN}$ training & 70 & $53 \%$ & $76.3(5.1)$ & $29.0(0.9)$ \\
\hline $\mathrm{CN}$ testing & 70 & $47 \%$ & $76.1(4.8)$ & $29.0(0.9)$ \\
\hline sMCI & 70 & $41 \%$ & $76.3(7.2)$ & $27.2(3.5)$ \\
\hline pMCI & 70 & $42 \%$ & $75.8(6.6)$ & $26.1(2.1)$ \\
\hline $\mathrm{AD}$ & 70 & $55 \%$ & $76.6(7.7)$ & $23.0(3.3)$ \\
\hline
\end{tabular}

bias during our experiments, we randomly selected subjects from each group with the constraint of similar age and similar gender proportion between groups. See Table 1 for details. As explained later, the CN (training) group is used to learn dictionaries (i.e., to create a sparse representation of the normal brain anatomy) while the testing $\mathrm{CN}$ group is used to model the inter-subject variability. All the data were preprocessed with the Statistical Parametric Mapping 8 software (SPM8; http://www.fil.ion.ucl.ac.uk/spm) and the VBM8-toolbox (http: //dbm.neuro.uni-jena.de/vbm.html) using default settings.

\subsection{Sparse-based modeling of the brain anatomy}

The proposed method estimates at each voxel the distribution of a scoring function measuring the degree of abnormality of a population at this location. The choice of the scoring function is the key to success for such an approach and thus it should be designed to be invariant to inter-subject variabilities inside the reference group while being discriminant to intra-group variabilities. In order to capture the local morphological structure of the brain, for each location in the MNI space, we use a 3D patch (i.e., a small rectangular windows) centered in a voxel $v$. The proposed scoring function measures the ability of this patch to be decomposed in a sparse way, i.e., as a linear combination of a small number of predefined patches. This family of predefined patches, called dictionary, is learned offline from the set of patches located at $v$ in the training group. In this study, we then distinguish three groups $G_{1}, G_{2}$ and $G_{3}$. The first group $G_{1}$ composed of $\mathrm{CN}$ subjects is used to learn the dictionary (CN training). Distributions of the scoring functions are next computed independently on two other groups, a reference group $G_{2}$ composed of $\mathrm{CN}$ subjects (CN testing) and the group of interest $G_{3}$ (e.g., composed of AD patients). Since $G_{1}$ and $G_{2}$ are two groups composed of $\mathrm{CN}$, the scoring function is expected to be statistically different for groups $G_{2}$ and $G_{3}$ where $G_{3}$ is abnormal compared to the control.

\subsection{Sparse brain anatomy representation using dictionary learning}

For each subject $i$ of group $G_{1}$ and at each voxel $v$ in the MNI space, a 3D patch is extracted, centered (subtraction of its mean), normalized (divided by its norm) and vectorized into a vector $x^{i}$. The dictionary $D$, that is a matrix whose columns are $p$ patches, is next selected as the one minimizing the following 
quantity:

$$
\min _{D, \alpha_{i}} \sum_{i=1}^{\left|G_{1}\right|} \frac{1}{2}\left\|x^{i}-D \alpha_{i}\right\|_{2}^{2}+\lambda\left\|\alpha_{i}\right\|_{1}
$$

where $\lambda$ is a positive parameter. The first term $\frac{1}{2}\left\|x^{i}-D \alpha_{i}\right\|_{2}^{2}$ ensures that each extracted patch $x^{i}$ is not too far from its approximation $D \alpha_{i}$, while the second term $\left\|\alpha_{i}\right\|_{1}$ promotes that each extracted patch $x^{i}$ can be indeed efficiently decomposed in a sparse way into the resulting dictionary $D$ (i.e., such that $\alpha_{i}$ contains a large number of zero coefficients). At the end of the procedure, each column of $D$ is a vectorization of a patch encoding one of the possible local morphological configurations of the brain in the training group $G_{1}$.

\subsection{Group comparison using sparse-based morphometry}

As for the dictionary learning step, a patch is extracted at voxel $v$ for each subjects of groups $G_{2}$ and $G_{3}$. This patch is also centered, normalized and vectorized. The following scoring function

$$
f(x)=\min _{\alpha \in \mathbb{R}^{p}} \frac{1}{2}\|x-D \alpha\|_{2}^{2}+\lambda\|\alpha\|_{1}
$$

is then applied on each patch where $D$ is the learned dictionary associated to voxel $v$. The empirical distribution of the scoring function $f(x)$ is next computed independently on each group. Thanks to the learning of an adapted dictionary on the CN training subjects of $G_{1}$, the dictionary $D$ is expected to be flexible enough to reconstruct sparsely most of the patches of the CN testing group $G_{2}$ used as reference, hence leading to statistically low scoring values $f(x)$. On the contrary, at locations where the group of interest $G_{3}$ presents some abnormalities, a low approximation error $\frac{1}{2}\|x-D \alpha\|_{2}^{2}$ might not be reachable with low sparsity level, hence leading to statistically large values of $\|\alpha\|_{1}$ and $f(x)$. After applying a T-test on the distributions of $f(x)$ estimated at each voxel, our sparse-based morphological technique provides a map of degree of difference between the groups $G_{2}$ and $G_{3}$. Low values in this map can next be used to determine the voxels where $G_{3}$ seems to present some abnormalities.

Fusion of SBM and VBM As usually done in sparse-based approaches, the patches are centered and normalized during the proposed SBM procedure. Therefore, the mean of the patches (i.e., the local GM density used for VBM) is not taken into account in SBM. We propose to fuse the SBM and VBM features in order to capture jointly the local anatomical geometry and the local tissue density. To this end, we propose to fuse the T-maps produced by SBM and VBM. The simple approach that takes the minimal p-value at each voxel is known to not produce p-values [8]. We use Bonferroni's method that is guaranteed to produce p-values from $K$ p-values $p_{k}$ using the fusion function $F$ defined as:

$$
F\left(p_{1}, \ldots, p_{K}\right):=K \min \left(p_{1}, \ldots, p_{K}\right) .
$$


Table 2: Abnormality ratios in $\%$ and merged p-values $(\tilde{p})$ in parentheses for each considered patch size. The presented results were obtained on $70 \mathrm{CN}$ vs. 70 AD. The used threshold on the T-maps was set to 0.01 .

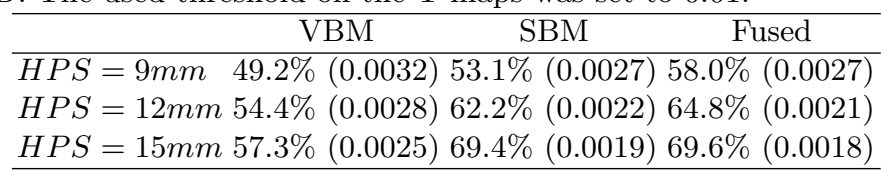

Table 3: Same as above but for each considered group size. The presented results were obtained on CN vs. AD using $H P S=15 \mathrm{~mm}$.

\begin{tabular}{lcc}
\hline VBM & SBM & Fused \\
\hline $30 \mathrm{CN}$ vs. $30 \mathrm{AD} 29.8 \%(0.0048)$ & $47.5 \%(0.0033)$ & $46.4 \%(0.0035)$ \\
$50 \mathrm{CN}$ vs. $50 \mathrm{AD} 57.9 \%(0.0028)$ & $65.4 \%(0.0023)$ & $67.3 \%(0.0021)$ \\
$70 \mathrm{CN}$ vs. $70 \mathrm{AD} 57.3 \%(0.0025)$ & $69.4 \%(0.0019)$ & $69.6 \%(0.0018)$ \\
\hline
\end{tabular}

Table 4: Same as above but for each considered testing group. The presented results were obtained with $H P S=15 \mathrm{~mm}$ and 70 subjects per group.

\begin{tabular}{lccc}
\hline & VBM & SBM & Fused \\
\hline SMCI & $0.1 \%(0.0170)$ & $1.3 \%(0.0132)$ & $0.3 \%(0.0147)$ \\
pMCI & $34.5 \%(0.0045)$ & $53.7 \%(0.0046)$ & $51.2 \%(0.0043)$ \\
AD & $57.3 \%(0.0025)$ & $69.4 \%(0.0019)$ & $69.6 \%(0.0018)$ \\
\hline
\end{tabular}

Implementation details and Quality metric In our experiments the value of $\lambda$ is not crucial and set to 0.15. The optimization of Eq. (1) is computed by alternating minimization on the dictionary $D$ and the sequence $\left(\alpha_{i}\right)$ using SPAMS' toolbox (http://spams-devel.gforge.inria.fr/index.htm) [9]. In the following, the half patch sizes (HPS) are reported in $\mathrm{mm}$. The number of atoms in the dictionary is set to 8 . This number does not have a drastic impact on results according to our experiments. All images are displayed using the same p-value range from $10^{-2}$ to $10^{-8}$ and at the same coordinates in the MNI space. The methods are evaluated with two metrics. First, the abnormality ratio metric estimates the percentage of GM detected as abnormal (i.e., with p-value inferior to 0.01$)$. Second, a merged p-value $(\tilde{p})$ metric reflects the level of significance in the area detected as abnormal. The p-values inferior to 0.01 are aggregated using the merging function [8] that produces a p-value (unlike the standard average):

$$
M\left(p_{1}, \ldots, p_{K}\right):=\frac{2}{K}\left(p_{1}+\ldots+p_{K}\right)
$$

\section{Results}

\subsection{Impact of the patch size and number of subjects}

Table 2 presents the abnormality ratio and the merged p-value $\tilde{p}$ obtained for the considered patch sizes. The abnormality ratio increases when using bigger patch sizes and the fusion of SBM and VBM lead to similar or higher ratios. In terms of significance, SBM and the fusion provides lower $\tilde{p}$ than VBM. In addition, the 
fusion of VBM and SBM produces lower $\tilde{p}$ than SBM alone. We can note that a plateau is reached at $H P S=15 \mathrm{~mm}$ for $\mathrm{VBM}$ and that the $\tilde{p}$ values are stable for SBM and the fusion. We therefore use this patch size in the following. Table 3 presents the abnormality ratio and the $\tilde{p}$ obtained for the considered group sizes. As expected, more significant differences are found between both groups when using a larger number of samples. Moreover, VBM is more affected by the reduction of the group size than SBM.

\subsection{Early detection of AD signature}

In this last experiment, we investigate the results provided by the methods on sMCI and pMCI groups. The pMCI group is expected to produce abnormalities close to the abnormalities detected in the AD group since the pMCI group is composed by patients converting to AD during the follow-up. The sMCI is expected to be closer to the $\mathrm{CN}$ group and can be viewed as an earlier stage of the AD compared to pMCI. Figure 1 shows the results of our abnormality detection approach on the considered groups (i.e., AD, pMCI, and sMCI). Table 4 presents the abnormality ratio and the $\tilde{p}$ obtained for each group. As expected, for all the methods, more abnormalities were detected in the AD group, followed by pMCI and sMCI. In all cases, SBM produces a higher abnormality ratio than VBM. Moreover, VBM produces a very low abnormality ratio for the sMCI group. These results seem to indicate that VBM is not sensitive enough to detect to subtle anatomical alterations present in the sMCI group. Consequently, the low abnormality ratio produced by VBM for the sMCI group impacts the fusion. For pMCI and AD groups similar results are obtained for SBM and fusion in terms of the abnormality ratio. However, fusion provides lower $\tilde{p}$ than SBM. In terms of anatomical difference, SBM finds significant differences for pMCI group in areas only detected for the AD group using VBM (e.g., medial frontal cortex and thalamus). VBM and SBM detect abnormalities in the posteriori cingulate cortex, while only SBM detects abnormality in the anterior cingulate cortex. Moreover, SBM found differences in the precuneus and the posterior cingulate cortex. Both structures have been shown to be impacted by AD in terms of anatomical atrophy [10, metabolism reduction [11] and functional alteration [12. In addition, SBM and VBM detect abnormalities in the left entorhinal and parahippocampal cortex for the sMCI group with a larger extentfor SBM. It is interesting to note that these structures are known to be affected at the first stage of the pathology [13. Finally, SBM is able to find subtle abnormalities in the medial frontal cortex in the sMCI group while such alterations are detected only in the AD group using VBM (see Fig. 1). This last result highlights the high sensitivity of SBM compared to VBM.

\section{Conclusion}

In this paper, we proposed a new method to detect anatomical abnormalities called sparse-based morphometry. Based on local modeling of the normal 
anatomy using dictionary learning, SBM enables us to efficiently capture brain alterations in terms of their geometry and shape. In our experiments, SBM was shown to be more sensitive than VBM when using small groups or when studying early anatomical modifications caused by AD at the first stage of the pathology. We also presented a fusion strategy to combine VBM and SBM in order to take into account modifications of the local average GM density and to capture alterations of the local shape of GM patterns.

\section{Acknowledgments}

This study has been carried out with financial support from the French State, managed by the French National Research Agency (ANR) in the frame of the Investments for the future Program IdEx Bordeaux (HL-MRI ANR-10-IDEX03-02), Clusters of excellence CPU and TRAIL (HR-DTI ANR-10-LABX-57) and the CNRS multidisciplinary project "Défi imag'In".

\section{References}

1. Ashburner, J., Friston, K.J.: Voxel-based morphometry - the methods. Neuroimage 11(6) (2000) 805-821

2. Shen, S., et al.: VBM lesion detection depends on the normalization template: a study using simulated atrophy. Magnetic resonance imaging 25(10) (2007) 13851396

3. Coupé, P., et al.: Patch-based segmentation using expert priors: Application to hippocampus and ventricle segmentation. NeuroImage 54(2) (2011) 940-954

4. Tong, T., et al.: Segmentation of MR images via discriminative dictionary learning and sparse coding: application to hippocampus labeling. NeuroImage 76 (2013) $11-23$

5. Coupé, P., et al.: Simultaneous segmentation and grading of anatomical structures for patient's classification: application to alzheimer's disease. NeuroImage 59(4) (2012) 3736-3747

6. Liu, M., et al.: Ensemble sparse classification of alzheimer's disease. NeuroImage 60(2) (2012) 1106-1116

7. others, B.W.: Standardization of analysis sets for reporting results from ADNI MRI data. Alzheimer's \&amp; Dementia 9(3) (2013) 332-337

8. Vovk, V.: Combining p-values via averaging. arXiv preprint arXiv:1212.4966 (2012)

9. Mairal, J., et al.: Online dictionary learning for sparse coding. In: 26th AICML, ACM (2009) 689-696

10. Karas, G., et al.: Precuneus atrophy in early-onset alzheimer's disease: a morphometric structural mri study. Neuroradiology 49(12) (2007) 967-976

11. Ikonomovic, M., et al.: Precuneus amyloid burden is associated with reduced cholinergic activity in alzheimer disease. Neurology 77(1) (2011) 39-47

12. He, Y., et al.: Regional coherence changes in the early stages of alzheimer's disease: a combined structural and resting-state functional mri study. Neuroimage 35(2) (2007) 488-500

13. Devanand, D., et al.: Hippocampal and entorhinal atrophy in mild cognitive impairment prediction of alzheimer disease. Neurology 68(11) (2007) 828-836 

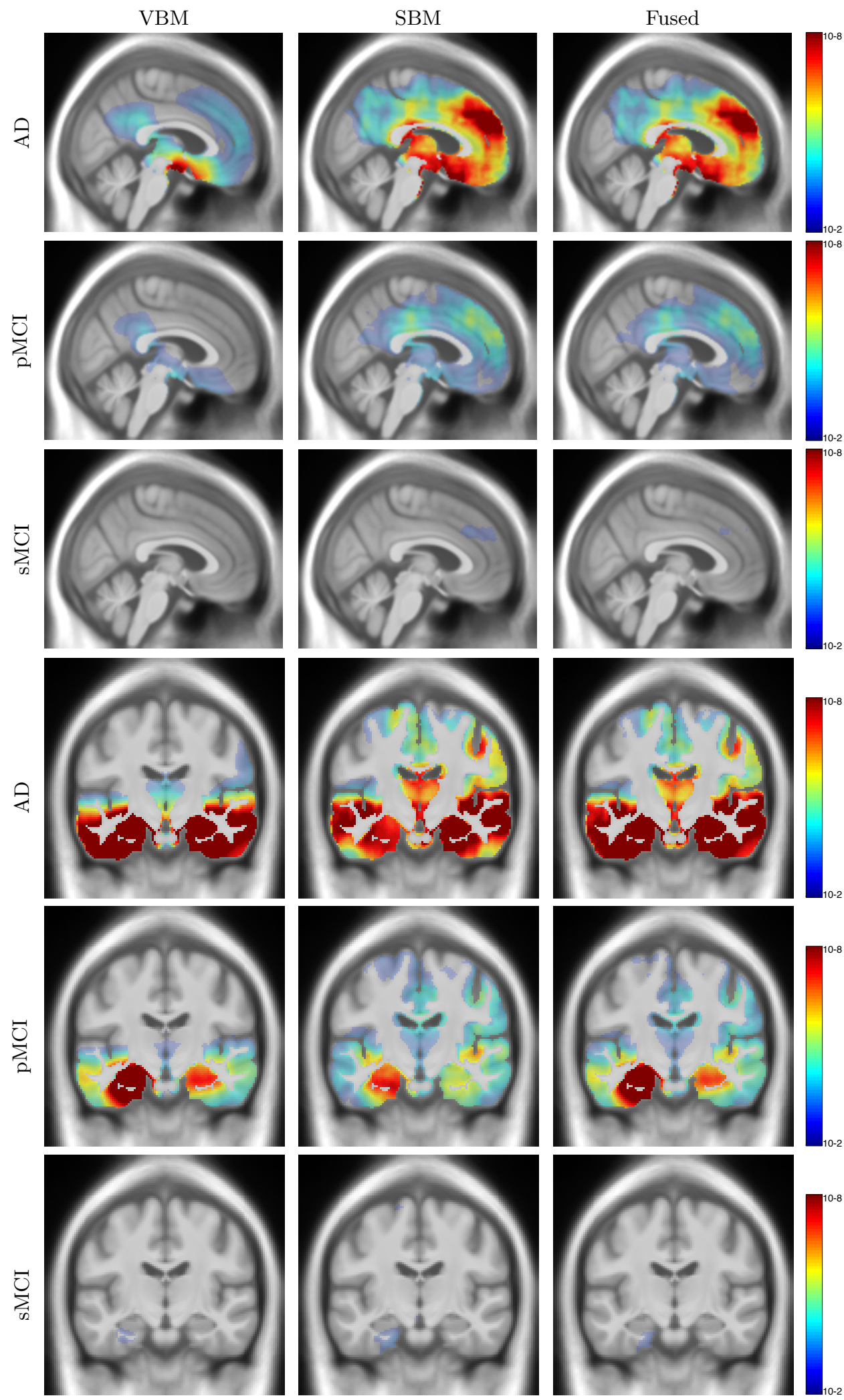

Fig. 1: Detection of abnormality in $\mathrm{AD}$, sMCI and pMCI groups using $H P S=15 \mathrm{~mm}$ and 70 subjects per group. The p-values of the T-maps are thresholded at 0.01 . 